\title{
Treatment Outcome for Head and Neck Squamous Cell Carcinoma in a Developing Country: University Malaya Medical Centre, Malaysia from 2003-2010
}

\author{
Yoke Fui Wong, Mastura Md Yusof, Wan Zamaniah Wan Ishak, Adlinda Alip, \\ Vincent Chee Ee Phua*
}

\begin{abstract}
Background: Head and neck cancer (HNC) is the eighth most common cancer as estimated from worldwide data. The incidence of HNC in Peninsular Malaysia was reported as 8.5 per 100,000 population. This study was aimed to determine the treatment outcomes for HNC patients treated in the Oncology Unit of University Malaya Medical Centre (UMMC). Materials and Methods: All newly diagnosed patients with squamous cell carcinoma of head and neck (HNSCC) referred for treatment to the Oncology Unit at UMMC from 2003-2010 were retrospectively analyzed. Treatment outcomes were 5-year overall survival (OS), cause specific survival (CSS), loco-regional control (LRC) and radiotherapy (RT) related side effects. Kaplan-Meier and log rank analyses were used to determine survival outcomes, stratified according to American Joint Committee on Cancer (AJCC) stage. Results: A total of 130 cases were analysed. Most cases $(\mathbf{8 1 . 5 \%})$ were at late stage (AJCC III-IVB) at presentation. The 5-year OS for the whole study population was $34.4 \%$ with a median follow up of 24 months. The 5-year OS according to AJCC stage was $100 \%, 48.2 \%, 41.4 \%$ and $22.0 \%$ for stage I, II, III and IVA-B, respectively. The 5-year overall CSS and LCR were 45.4\% and 55.4\%, respectively. Late effects of RT were documented in $41.4 \%$ of patients. The most common late effect was xerostomia. Conclusions: The treatment outcome of HNSCC at our centre is lagging behind those of developed nations. Efforts to increase the number of patients presenting in earlier stages, increase in the use of combined modality treatment, especially concurrent chemoradiotherapy and implementation of intensity modulated radiotherapy, may lead to better outcomes for our HNC patients.
\end{abstract}

Keywords: Squamous cell carcinoma of head and neck - treatment outcome - radiotherapy

Asian Pac J Cancer Prev, 16 (7), 2903-2908

\section{Introduction}

Head and neck cancer (HNC) is the eighth most common cancer as estimated from the worldwide incidence in 2008 (Jacques et al., 2008). Squamous cell carcinoma (SCC) is the predominant histology, involving almost 90$95 \%$ of all HNC cases. Two thirds of the cancer burden is borne by the developing countries where squamous cell carcinoma of the head and neck (HNSCC) region is one of the leading causes of cancer mortality. In South East Asia, HNC accounts for approximately 8-10\% of all cancers. The incidence of HNC in Peninsular Malaysia was reported as 8.5 per 100,000 population (National Cancer Registry, Malaysia, 2006) which is considerably higher than the average global incidence in both developed $(6.9$ per 100,000$)$ and less developed regions (4.6 per $100,000)$ (Jemal et al., 2011). The prognosis and survival of patients with HNC are dependent on disease stage at presentation and the quality of care provided to each patient. In Malaysia, the majority of patients with HNSCC classically present late, with locally advanced disease, defined as stage III or non-metastatic stage IVA-B. In a retrospective study on the treatment outcome of 677 patients treated for HNC over 10 years in a tertiary hospital in the northern region of Malaysia, nearly $80.5 \%$ of the patients presented with locally advanced stage (Shashinder et al. 2008). The diagnosis of locally advanced HNSCC confers a poor prognosis with 5-year survival rates of only about $10-40 \%$.

Early to locally advanced stage HNSCC can be treated with surgery, radiation (with or without chemotherapy) or a combination of both. The superiority of combined multimodality treatment versus either surgery or radiotherapy (RT) alone in improving the treatment outcome of HNSCC have been demonstrated in various large clinical trials (Brownan et al., 2001; Pignon et al., 2009). Conventional once daily RT in 1.8-2Gray (Gy) per fraction to a total of 66-70Gy in 6.5-7 weeks given concomitantly with platinum-based chemotherapy is the gold standard for locally advanced HNSCC with the aims 
of optimising loco-regional control (LRC) and survival while preserving organ function whenever possible. This results in high LRC rate of $50-60 \%$ at 2 years and overall survival (OS) rate of approximately $50 \%$ at 5 years (Gupta et al., 2014). A large survival analysis study of 5595 HNC patients treated with conventional RT between 1987-89 in Mumbai reported a 5-year OS in the range of 20-43\% for oral cavity cancers, $8-25 \%$ for pharyngeal cancers and 25-62\% for laryngeal cancers (Rao et al., 1998). A recent paper from the South East Asia revealed a low 5-year OS of only $24.1 \%$ (Pruegsanusak et al., 2012).

Concurrent chemoradiotherapy (CCRT) provides superior outcome but at the expense of higher toxicity than RT alone. Other factors that may affect the treatment delivery and outcome are patient's comorbidities, performance status, nutritional status and habits such as cigarette smoking and alcohol intake. A Radiation Therapy Oncology Group (RTOG) analysis of 230 patients treated with CCRT showed that approximately $43 \%$ of assessable patients had severe late toxicity like dysfunction of the larynx and/or pharynx (eg. dysphagia). In this study, older age, advanced tumour stage, and larynx/hypopharynx primary site were strong independent risk factors for severe late toxicity (Mitchell et al., 2008).

This study was taken to analyse in a retrospective manner, the treatment outcome of patients with HNSCC treated in Oncology unit at University of Malaya Medical Centre (UMMC), a tertiary academic hospital in Malaysia with the primary endpoints of 5-year OS and secondary endpoints of 5-year cause specific survival (CSS) and 5-year LRC rates.

\section{Materials and Methods}

Data collection was initiated following institutional Ethnics Committee approval. A retrospective review on all HNSCC patients treated in the unit of clinical oncology, UMMC between January 2003-December 2010 were carried out. All previously untreated stage I to IVB patients who have histology-proven SCC arising from the oral cavity, oropharynx, larynx and hypopharynx were selected as subjects for this study. Patients who had received neoadjuvant chemotherapy were included. Patients without histological confirmation, presenting with recurrence or metastases, having a non SCC histology, carcinoma of unknown primary, salivary gland carcinoma and patients who received treatment at other oncology centres were excluded from the analysis. Patients who did not complete their planned RT or chemotherapy schedules were included to avoid attrition bias and to reflect the reality of our local clinical setting.

Information collected included patient demographics, clinical stage based on tumour, lymph nodes and distant metastasis (TNM) and American Joint Committee on Cancer (AJCC) staging for HNC of 6th edition, treatment received which included surgery, radiotherapy, any neoadjuvant, concurrent or adjuvant chemotherapy and treatment outcome. Treatment outcome of 5-year OS, 5-year CSS, 5-year LRC and incidence of late side effects were determined.

Overall survival was defined as the time interval from the date of diagnosis to the date of death from any cause. Cause specific survival was defined as the time interval from the date of diagnosis to the date of death due to HNC. Loco-regional control was defined as the time interval from the date of diagnosis to the date of loco-regional relapse. Patients lost to follow-up were contacted via phone to determine their current status while the status of non-contactable patients was retrievedfrom the National Registration Department (NRD). Statistical analysis was carried out using the SPSS v.18 software. Kaplan-Meier and log rank analysis was used to determine survival outcomes, which was stratified according to AJCC stage.

\section{Results}

Between $1^{\text {st }}$ January 2003 and $31^{\text {st }}$ December 2010, a total of 130 patients with oral cavity, oropharynx, larynx and hypopharynx carcinoma were eligible for this study. The clinicopathological features and treatment received by the patients are listed in Table 1. Majority of patients were within the 51-69 years age group (53.8\%) with mean age of 63.3 years (ranged $31-85$ years). Almost two thirds $(63.8 \%)$ were males. Chinese was the predominant race $(45.4 \%)$, followed by Malay $(35.4 \%)$ and Indian $(18.5 \%)$. Majority of the patients had good performance status between 0 and $1(83.8 \%)$ and comorbidities such as hypertension, diabetes mellitus and cardiac disease were present in nearly half of the patients $(45.4 \%)$. Oral cavity was the predominant site of tumor accounting for $49.2 \%$, followed by larynx at $33.8 \%$. Late presentation was commonly observed in our patient population with $67.6 \%$ with T3-4 disease, $58.5 \%$ with N1-3 nodal involvement and $81.5 \%$ with late stage disease(AJCC III-IVB).

Eighty-two patients $(63.1 \%)$ received RT as the primary treatment. Forty-eight patients $(36.9 \%)$ had RT alone and 34 patients $(26.2 \%)$ had CCRT. Out of 48 patients $(36.9 \%)$ who had primary surgery, 34 patients (70.8\%) and 8 patients $(16.7 \%)$ received adjuvant RT and CCRT respectively. The remaining six patients had surgery alone. Approximately $76.9 \%$ completed RT within seven weeks. Only 6 patients $(4.6 \%)$ received neoadjuvant chemotherapy and no patient received adjuvant chemotherapy.

With a median follow up of 24 months, the 5-year OS for the whole study population was $34.4 \%$.The 5-year OS according to AJCC stage was $100 \%, 48.2 \%, 41.4 \%$ and $22.0 \%$ for stage I, II, III andIVA-B respectively (Figure 1). The median survival for AJCC stage IVA-B was 11.5 months. The oropharynx $(n=12)$ subsite demonstrated the highest 5-year OS with $41.7 \%$, followed by larynx $(n=44)$ with $40.9 \%$. Five years OS for oral cavity $(n=64)$ was $25 \%$ while the rate for hypopharynx $(n=10)$ was $10 \%$.

Ninety deaths had occurred at the time of study analysis. Out of 90 deaths, 71 were due to HNSCC while the causes of other deaths were old age $(n=6)$, pneumonia $(n=3)$, septic shock $(n=2)$, acute myocardiac infarction $(n=1)$, upper gastrointestinal bleeding $(n=1)$, carcinoma of lung $(n=1)$, chronic lung disease $(n=1)$ and uncertain cause $(n=4)$. The overall 5-year CSS was $45.4 \%$ while the 5-year CSS according to stage were 100\%, 68.8\%, 48.3\% and $33.8 \%$ for stages 1 , II, III and IVA-B respectively 
(Figure 2).

The overall 5-year LRC rate was 55.4\%. The 5-year LRC according to stage were $100 \%$ for stage $1,62.5 \%$ for stage II, $55.2 \%$ for stage III and $49.4 \%$ for stage IVA-B (Figure 3).Seventy-four recurrences (56.9\%) were recorded with predominance of loco-regional recurrence over distant metastases. The commonest pattern was loco-regional recurrence $(19.2 \%)$ followed by regional recurrence $(16.2 \%)$ and distant metastasis $(12.3 \%)$. The least common was local recurrence $(9.2 \%)$. Stage IVA-B subgroup developed the most recurrence with $19.5 \%$ developing distant metastases (15/77) and $18.2 \%$ (14/77) recurring loco-regionally. Patients with stage III disease who recurred developed mostly loco-regional

Table 1. Clinicopathological Features and Outcome of 130 Patients

\begin{tabular}{|c|c|c|c|}
\hline & Item & $\begin{array}{l}\text { No. of } \\
\text { Patients }\end{array}$ & $\%$ \\
\hline \multirow[t]{3}{*}{ Age (years) } & $<50$ & 20 & 15.4 \\
\hline & $51-69$ & 70 & 53.8 \\
\hline & $\geq 70$ & 40 & 30.8 \\
\hline \multirow[t]{2}{*}{ Gender } & Male & 83 & 63.8 \\
\hline & Female & 47 & 36.2 \\
\hline \multirow[t]{4}{*}{ Race } & Malay & 24 & 18.5 \\
\hline & Chinese & 59 & 45.4 \\
\hline & Indian & 46 & 35.4 \\
\hline & Others & 1 & 0.8 \\
\hline \multirow[t]{4}{*}{ Performance Status } & 0 & 44 & 33.8 \\
\hline & 1 & 65 & 50 \\
\hline & 2 & 16 & 12.3 \\
\hline & 3 & 5 & 3.8 \\
\hline \multirow{2}{*}{ Co-morbidities } & Yes & 59 & 45.4 \\
\hline & No & 71 & 54.6 \\
\hline \multirow[t]{4}{*}{ Primary Tumor Site } & Oral Cavity & 64 & 49.2 \\
\hline & Oropharynx & 12 & 9.2 \\
\hline & Larynx & 44 & 33.8 \\
\hline & Hypopharynx & 10 & 7.7 \\
\hline \multirow[t]{4}{*}{ Tumor Stage } & $\mathrm{T} 1$ & 12 & 9.2 \\
\hline & $\mathrm{T} 2$ & 30 & 23.1 \\
\hline & $\mathrm{T} 3$ & 31 & 23.8 \\
\hline & $\mathrm{T} 4$ & 57 & 43.8 \\
\hline \multirow[t]{4}{*}{ Nodal Stage } & No & 54 & 41.5 \\
\hline & N1 & 15 & 11.5 \\
\hline & $\mathrm{N} 2$ & 49 & 37.7 \\
\hline & $\mathrm{N} 3$ & 12 & 9.2 \\
\hline \multirow[t]{4}{*}{ AJCC Stage } & I & 8 & 6.2 \\
\hline & II & 16 & 12.3 \\
\hline & III & 29 & 22.3 \\
\hline & IV & 77 & 59.2 \\
\hline \multirow[t]{3}{*}{ Mode of Treatment } & EBRT alone & 48 & 36.9 \\
\hline & CCRT & 34 & 26.2 \\
\hline & Surgery & 48 & 36.9 \\
\hline Overall Treatment time (OTT) & Within 7 weeks & 93 & 76.9 \\
\hline \multirow[t]{2}{*}{ Neoadjuvant Chemotherapy } & $>7$ weeks & 28 & 23.1 \\
\hline & Yes & 6 & 4.6 \\
\hline \multirow[t]{2}{*}{ Late Effects } & No & 124 & 95.4 \\
\hline & Yes & 51 & 41.1 \\
\hline \multirow[t]{2}{*}{ Recurrence } & No & 73 & 58.9 \\
\hline & Yes & 74 & 56.9 \\
\hline \multirow[t]{5}{*}{ Site of Recurrence } & No & 56 & 43.1 \\
\hline & Local & 12 & 9.2 \\
\hline & Regional & 21 & 16.2 \\
\hline & Locoregional & 25 & 19.2 \\
\hline & Distant & 16 & 12.3 \\
\hline
\end{tabular}

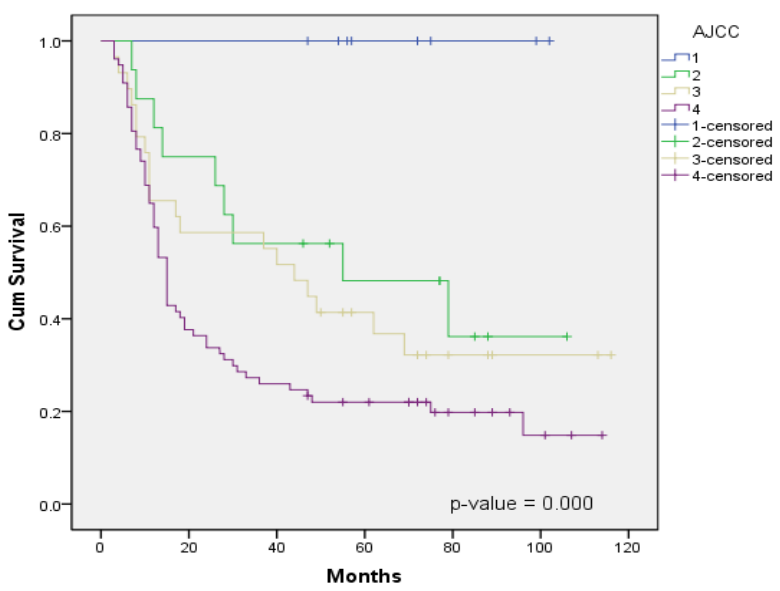

Figure 1. Overall Survival According to AJCC Stage

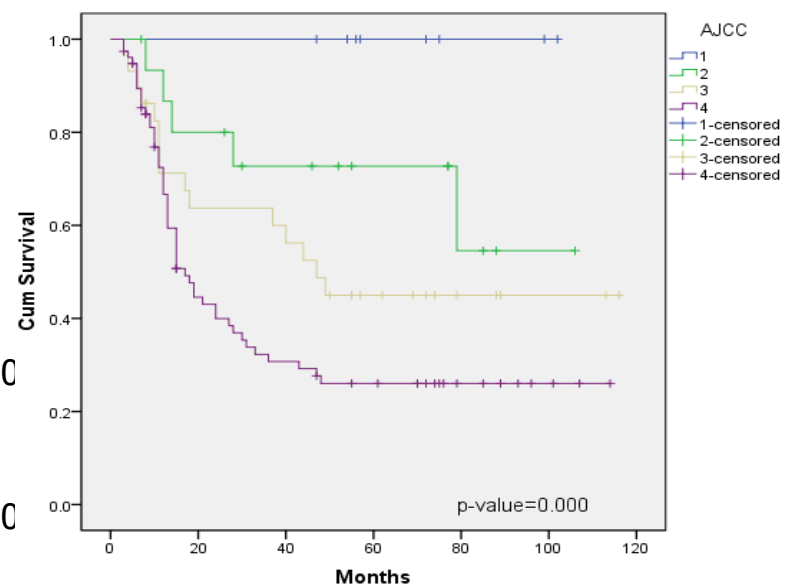

Figure 2. Cause Specific Survival According to AJCC Stage

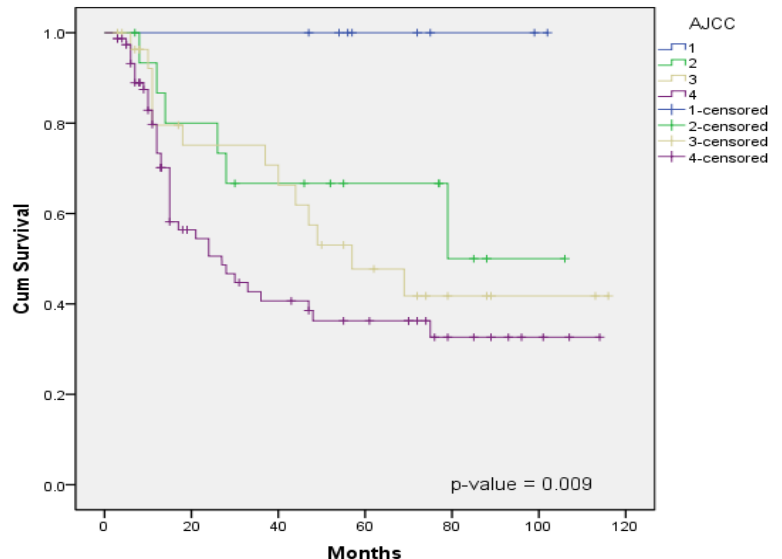

Figure 3. Loco-regional Control According to AJCC Stage

disease $(20.7 \%, 6 / 29)$. Only 2 patients $(6.7 \%)$ in this stage developed distant metastasis.

Late effects of RT were documented in $41.1 \%$ of patients who received RT in this study $(n=124)$. The most common late effect was xerostomia. Other late effects documented were neck stiffness, dysphagia, trimus and hoarseness of voice.

\section{Discussion}

In the present study, new cases of HNSCC diagnosed during 2003 to 2010 were analyzed for their 5-year 
treatment outcome and associated clinicopathological variables. A full understanding of regional cancer epidemiology is an important basis for determining the strategy for absolute cancer control and future research. The mean age of 63.3 years and men being the predominant gender as observed in our study correlates with the Asian epidemiology for head and neck cancer (Bhurgri et al., 2006; Lasrado et al., 2012). Furthermore, oral cavity and larynx were similarly the most commonly affected site as compared to pharynx in our study and the above study (Bhurgri et al., 2006).

The main result of this series showed a 5-year OS of $34.4 \%$. This survival rate is considerably lower compared to results obtained from Western countries (Barzan et al., 2002; Carvalho et al., 2009; MacKenzie et al., 2009) but is similar to a study from a neighbouring country, Thailand (Pruegsanusak et al., 2012). There are, several possible explanations for the discrepancies. Surgery alone as a single modality of treatment is a standard treatment for stage I or early stage II disease. The majority of patients $(81.5 \%)$ in this study present at an advanced stage (stage III to IVB) at diagnosis precluding treatment with surgery only. In our study, the surgery alone rate $(4.6 \%)$ is remarkably low compared to other series like the series of SEER of the US (10.2\%-48.9\%). Advanced stage at presentation were largely noted in epidemiological studies done in developing or low income countries such as India (Mohanti et al., 2007) and Brazil (De Paula et al., 2009). This is in contrast with lower stage disease at presentation predominantly seenin Western countries (Rusthoven et al., 2008; MacKenzie et al., 2009). Multiple factors may contribute to advanced stage at presentation, including personal factors, health education, health care access and low socioeconomic status. The high proportion of late presentation in our study $(81.5 \%)$ can partially explain our low survival rate.

Although the prognosis and survival of patients with $\mathrm{HNC}$ are dependent on disease stage at presentation, the quality of care provided an impact on the overall outcome of each patient. The overall diagnostic process, assessment of the patient, and differences in the availability and quality of oncology surgery, radiotherapy and medical oncology services may impact treatment selections and outcome. Other additional factors such as patient's comorbidities, socioeconomic factors as well as educational background also play a role. Most large randomized trials were conducted in a controlled research setting primarily in developed countries, involving selected groups of patients. Furthermore, existing clinical guidelines for the management of HNC have been usually developed in and for the affluent western world where modern resources for optimum care are available at all times. Different patient profile in the developing countries such as poorer nutrition, performance status, renal function or prior anaemia and limited resources for handling of toxic effects could lead to a poorer tolerance of more aggressive regimens such as CCRT or postoperative RT. The vast majority of these clinical evidence establishing this approach were acquired from developed countries that are better equipped and capable of delivering more intensive treatment schedules with appropriate supportive measures for treatment toxicities.

In this study, only $33.9 \%(42 / 124)$ of patients who had RT received CCRT. This might have contributed to a lower survival rate. The Meta-Analysis of Chemotherapy in Head and Neck Cancer (MACH-NC) with an updated result of 93 randomised trials with 17,346 patients showed benefit of concomitant chemotherapy achieving $8 \%$ improvement in OS rate at 5 years when compared to RT alone (Pignon et al., 2009). The benefit of CCRT in patients with advanced $\mathrm{HNC}$ and in patients requiring organ preservation was confirmed in several large randomized trials (Alelstein et al., 2003; Forastiere et al., 2003; Denis et al., 2004). However, the addition of chemotherapy to radiotherapy in older patients may be less effective and careful selection of patient are important in this setting (VanderWalde et al., 2014). We noted that $30.8 \%$ of our patients were in the above 70 years old age group and this may have resulted in the choice of RT alone.

Chemotherapy in the neoadjuvant setting has also been tested extensively in conjunction with standard therapy in the treatment of HNC. In the MACH-NC meta-analysis (Pignon et al., 2009), neo-adjuvant chemotherapy did not show any statistically significant effect on OS compared with surgery and/or RT alone (HR 0.96, 95\%CI 0.90-1.02) based on 31 trials with 5311 patients. However, when results were analyzed based on type of neo-adjuvant chemotherapy regimen used, OS with cisplatin plus 5-fluorouracil was significantly better than with surgery and/or RT alone (HR 0.90, 95\%CI 0.82-0.99), whereas there was no difference for other regimens or single agent chemotherapy. Newer randomized trials of neoadjuvant chemotherapy using combination of taxane, cisplatin and fluorouracil has shown further improved response rate and OS in locally advanced head and neck cancer (Pointreau et al., 2009; Jochen et al., 2011; Pierre et al., 2013). However, it was also associated with increased toxicity like neutropenic fever and a significant reduction in concurrent chemotherapy dose intensity had to be applied (Sanders et al., 2014). In our study, the number of patients having neo-adjuvant chemotherapy was low (4.6\%) despite many patients having advanced stage disease at presentation. This could be due to the perceived increased toxicity of neo-adjuvant chemotherapy and lower tolerance of Asian population to such regimen. The other commonly encountered problem with neo-adjuvant chemotherapy in this part of the world is the high rate of default after good response to initial treatment (Azrif et al., 2011). No patients in our study received adjuvant chemotherapy. There is no evidence to support the usage of adjuvant chemotherapy at this point of time and the MACH-NC meta-analysis which included six trials with 2567 patients on adjuvant chemotherapy showed no improvement in overall survival compared with definitive local therapy alone (HR 1.06, 95\%CI 0.95-1.16) and the results was not significant.

Radiotherapy delivery technique has been shown to affect treatment outcome. Patients in this study received radiotherapy either conventionally or with 3D conformal technique (3DCRT) due to non-availability of intensity modulated radiotherapy (IMRT) at this centre during the time period of this study. Overall survival and LCR results 
were lower when compared to the latest reported results using IMRT which had comparable rates of advanced stage patients. In these study, 2-year OS ranged around 77 to $87 \%$ (Traynor et al., 2010; Vila Capel et al., 2012) and 3-year OS of $60.3 \%$ and LCR of $66.1 \%$ (Van Gestel et al., 2011). IMRT, which provides highly conformal dose distribution, is rapidly gaining acceptance amongst radiation oncologists as a new approach in treating HNC. Usage of IMRT for HNC in Malaysia remains sparse. The implementation of IMRT in treating head and neck cancer at our institution is highly awaited.

The effect of OTT to treatment outcome is of great concern for head and neck carcinoma. In this study, only $76.9 \%$ of patients completed their RT within 7 weeks. Treatment time prolongation can have a deleterious effect on local tumor control and patient outcomes (Cannon et al., 2013). Among the possible causes of the prolonged OTT were planned unit maintenance and servicing, unexpected malfunction of linear accelerators and severe side effects of RT requiring breaks during the RT period. Patients who received RT and CCRT should be managed aggressively to limit treatment interruptions.

Late radiation effects were documented in $41.4 \%$ of our patient population though we are mindful that this is probably an underestimate as this is aretrospective study. Xerostomia was the most common late RT effect followed by neck stiffness, dysphagia and trismus. Dirix and colleagues (2008) reported that up to $93 \%$ of patients suffered from dry mouth and grade 2 to 3 xerostomia affected $65 \%$ of patients who responded to questionnaire in head and neck cancer life. These sequelae suggest that novel approaches are needed to minimize their severity. Parotid gland sparing IMRT for HNC patients improves xerostomia related quality of life compared to conventional radiotherapy (van Rij et al., 2008, Nutting et al., 2011). Although, hypothyroidism was not documented in this study it is a frequent late side effect of head and neck radiation and lifelong monitoring of thyroid function might be needed (Bernat et al., 2014). We expect implementation of IMRT for HNC at our centre will improve treatment outcome and reduce late toxicity especially xerostomia.

In summary, the treatment outcome of HNSCC at our centre is lagging behind those of developed nations. Efforts to increase the proportion of patients presenting in earlier stages, increase in the use of combined modality treatment especially CCRT and implementation of IMRT should lead to better outcomes for our HNC patients.

\section{References}

Adelstein DJ, Li Y, Adam GL, et al (2003). An intergroup phase III comparison of standard radiationtherapy and two schedules of concurrent chemoradiotherapy in patients with unresectable squamous cell head and neck cancer. J Clin Oncol, 21, 92-8.

Azrif M, Jalai I, Aslan NM, et al (2011). Neoadjuvant chemotherapy for locally advanced breast cancer in a Malaysian tertiary hospital. Asian Pac J Cancer Prev, 12, 157-62.

Barzan L, Talamini R, Franchin G, et al (2002). Changes in presentation and survival of head and neck carcinoma in
Northeastern Italy, 1975-1998. Cancer, 95, 540-52.

Bernat L, Hrusak D, et al (2014). Hypothyroidism after radiotherapy of headand neck cancer. $J$ Carniomaxillofaci Surg, 42, 356-61.

Bhurgri Y, Bhurgri A, Usman A, et al (2006). Epidemiological review of head and neck cancers in Karachi. Asian Pac J Cancer Prev, 7, 195-200.

Browman GP, Hodson DI, Mackenzie, et al (2001). Cancer care ontario practice guideline initiative head and neck cancer disease site group. choosing a concomitant chemotherapy and radiation therapy regimen for squamous cell head and neck cancer: a systematic review of the published literature with subgroup analysis. Head Neck, 23, 579-89.

Carvalho AL, Nishimoto IN, Califano JA, et al (2005). Trends in incidence and prognosis for head and neck cancer in the United States: a site-specific analysis of the SEER database. Int J Cancer, 114, 806-16.

Cannon DM, Geye HM, Hartig GK, et al (2013). Increased local failure risk with prolonged radiation treatment time in head and neck treated with concurrent chemotherapy. Head Neck, 36, 1120-5.

Daly T, Poulsen MG, Denham JW, et al (2003). The effect of anaemia on efficacy andnormal tissue toxicity following radiotherapy for locally advanced squamous cell carcinoma of the head and neck. Radiotherapy Oncol J Eur Soc Therapeutic Radiology Oncol, 68, 113-22.

Denis F, Garaud P, Bardet E, et al (2004). Final results of the 94-01 french head and neck oncology and radiotherapy group randomized trial comparing radiotherapy alone with concomitant radiochemotherapy in advanced stage oropharynx carcinoma. J Clin Oncol, 22, 69-76.

De Paula AM, Souza LR, Farias LC, et al (2009). Analysis of 724 cases of primary head and neck squamous cell carcinoma (HNSCC) with a focus on young patients and p53 immunolocalization. Oral Oncol, 45, 777-82.

Dirix P, Nuyts S, Vander Poorten V, et al (2008). The influence of xerostomia after radiotherapy on quality of life: results of a questionnaire in head and neck cancer. Supportive Care Cancer, 16, 171-9.

Forastiere AA, Goepfert H, Maor M, et al (2003). Concurrent chemotherapy and radiotherapy for organ preservation in advanced larnyngeal cancer. New England J Med, 349, 2091-8.

Furness S, Glenny AM, Worthington HV, et al (2011). Interventions for the treatment of oral cavity and oropharyngeal cancer: Chemotherapy (Review). Cochrane Database Syst Rev, 13, CD006386.

Gupta S, Weidong K, Christopher MB, et al (2014). Impact of concomitant chemotherapy on outcomes of radiation therapy for head and neck cancer: a population based study. Int $J$ Radiat Oncol Biol Phys, 88, 115-21.

Jacques F, Hai-Rim S, Freddie B, et al (2008). Estimates of worldwide burden of cancer in 2008: GLOBOCAN 2008. Int J Cancer, 127, 2893-010.

Jemal A, Bray F, Center MM, et al (2011). Global cancer statistics. CA Cancer J Clin I, 61, 69-90.

Jochen HL, Olga G, Robert IH, et al (2011). Induction chemotherapy with cisplatin and fluorouracil alone or in combination with docetaxel in locally advanced squamous cell cancer of the head and neck: long term results of TAX 324 randomised phase 3 trial. Lancet Oncol, 12, 153-9.

Lasrado S, Prabhu P, Kakria A, et al (2012). Clinicopathological profile of head and neck cancers in the Western development region, Nepal: a 4-year snapshot. Asian Pac J Cancer Prev, 13, 6059-62.

MacKenzie K, Savage SA, Birchall MA (2009). Processes and outcomes of head and neck cancer patients from 
geographically disparate regions of the UK. a comparison of Scottish and English cohorts. Eur J SurgOncol, 35, 1113-8.

Michell M, Jennifer M, Andrew T, et al (2008). Factors associated with severe late toxicity after concurrent chemoradiation for locally advanced head and neck cancer: an RTOG analysis. J Clin Oncol, 32, 3582-9.

Mohanti BK, Nachiappan P, Pandey RM, et al (2007). Analysis of 2167 head and neck cancer patients' management, treatment compliance and outcomes from a regional cancer centre, Delhi, India. J Laryngol Otol, 121, 49-56.

National Cancer Registry, Ministry of Health Malaysia (2006). Malaysian cancer statistics-data and figure peninsular Malaysia 2006.

Nutting CM, Morden JP, Harrington KJ, et al (2011). Parotid-sparing intensity modulated versus conventional radiotherapy in head and neck cancer (PARSPORT): a phase 3 multicentre randomized controlled trial. Lancet Oncol, 12, 127-36.

Pierre B, Jean B, Benjamin L, et al (2013). Taxane-cisplatin fluorouracil as induction chemotherapy in locally advanced head and neck cancers: an individual patient data metaanalysis of the meta-analysis of chemotherapy in head and neck cancer group. J Clin Oncol, 31, 2854-60.

Pignon JP, Le Maitre A, Maillard E, et al (2009). Meta-analysis of chemotherapy in head and neck cancer (MACH-NC): an update on 93 randomised trials and 17,346 patients. Radiother Oncol, 92, 4-14.

Pointreau Y, Garaud P, Chapet S, et al (2009). Randomized trial of inductionchemotherapy with cisplatin and 5-fluorouracil with or without docetaxel for larnynxpreservation. $J$ Nat Cancer Inst, 101, 498-506.

Pruegssanusak K, Peereavut S, Leelamanit V, et al (2012). Survival and prognosticprognostic factors of different sites of head and neck cancer: an analysis from Thailand. Asian Pac J Cancer Prev, 13, 885-9.

Rao DN, Shroff PD, Chattopadhyay G (1998). Survival analysis of 5595 head and neck cancer-results of conventional treatment in a high-risk population. Br J Cancer, 77, 1514-8.

Rusthoven K, Ballonoff A, Raben D, et al (2008). Poor prognosis in patients with stage I and II oral tongue squamous cell carcinoma. Cancer, 112, 345-51.

Sanders IW, Haslett K, Correa P, et al (2014). Sequential TPF

chemotherapy followed byconcurrentchemoradiotherapy in locally advanced head and neck cancer-a retrospective analysis of toxicity and outcomes. Scottish Med J, 59, 50-5.

Shashinder S, Choo PK, Gopala KG, et al (2008). Outcome of patients with head and neck cancers: 10-year experience of a otorhinolaryngology-head and neck unit in a tertiary hospital of a developing country. Eur J Cancer Care, 17, 93-7.

Traynor AM, Richards GM, Harting GK, et al (2010). Comprehensive IMRT plus weeklycisplatin for advanced head and neck cancer: the university of Wisconsin experience. Head Neck, 32, 599-606.

VanderWalde NA, Meyer AM, Deal AM, et al (2014). Effectiveness of chemoradiation for head and neck cancer in an older patient population. Int J Radiat Oncol Biol Phys, 89, 30-7.

Van Gestel D, Van Den WD, Schrijvers D, et al (2011). IntensityModulated Radiotherapy in patients with head and neck cancer: a Europeon single-centre experience. $\mathrm{Br} J$ Radiol, 84, 367-74.

Van Rij CM, Oughlane-heemsbergen WD, Ackerstaff AH, et al (2008). Parotid gland sparing IMRT for head and neck cancer improves xerostomia related quality of life. Radiat Oncol, 3, 41.

Vila Capel A, Vilar PJ, Pedro OA, et al (2013). IMRT: preliminary results in a series of advanced head and neck 